\title{
Status Quo der internetbasierten Nachhaltigkeitsberichterstattung
}

\section{Eine länderübergreifende Analyse der Nachhaltigkeits- berichte börsennotierter Unternehmen}

\author{
Michael Freundlieb, Frank Teuteberg \\ Fachgebiet Unternehmensrechnung und Wirtschaftsinformatik, \\ Universität Osnabrück
}

\section{Einleitung und Motivation}

Nachhaltigkeitsberichterstattung zielt darauf ab, gegenüber internen und externen Stakeholdern (Anspruchsgruppen) Rechenschaft darüber abzulegen, inwiefern das berichtende Unternehmen ökonomische, ökologische und soziale Nachhaltigkeitsziele miteinander in Einklang bringt, und welche Maßnahmen zur Verbesserung der Umweltleistung des Unternehmens ergriffen werden (Günther 2008, S. 333 u. 342 ff.). Wie eine aktuelle Befragung unter den Lesern von Nachhaltigkeitsberichten (NB) zeigt, werden NB häufig zur Entscheidungsunterstützung vor Kauf- oder Investitionsentscheidungen durch Konsumenten und potentielle Shareholder zu Rate gezogen (KPMG 2008 S. 9).

Neben der dadurch entstehenden Möglichkeit, sich durch Nachhaltigkeitsberichterstattung einen Wettbewerbsvorteil zu verschaffen, drängt auch der Gesetzgeber Unternehmen zunehmend zur Veröffentlichung von Nachhaltigkeitsinformationen. Ein Beispiel hierfür ist das Bilanzreformgesetz aus dem Jahre 2006, welches von großen Kapitalgesellschaften fordert, dass sie im Lagebericht Aussagen zu nichtfinanziellen Indikatoren machen, die Einfluss auf den Unternehmenserfolg haben (IÖW 2007, S. 12). Zusätzlich wächst auch durch das gesteigerte Interesse der Medien, den Erwartungen der Öffentlichkeit und Branchenvereinbarungen wie bspw. des European Chemical Industrial Council der Druck auf Unternehmen, einen NB zu veröffentlichen.

NB sind somit ein wichtiger Bestandteil der Unternehmenskommunikation, der durch den Einsatz von Informations- und Kommunikationstechnologie wirkungsvoll unterstützt werden kann. 
Dieser Beitrag zielt darauf ab, auf der Basis einer explorativen Analyse der Nachhaltigkeitsberichte von 97 börsennotierten Unternehmen den Status Quo und Best Practices der internetbasierten Nachhaltigkeitsberichterstattung zu ermitteln sowie darauf aufbauend zukünftigen Forschungsbedarf sowie Implikationen für Wissenschaft und Unternehmenspraxis abzuleiten.

Der Beitrag ist wie folgt aufgebaut: In Abschnitt 2 werden zunächst verwandte Arbeiten diskutiert. Die dieser explorativen Studie zugrundeliegende Vorgehensweise wird in Abschnitt 3 erläutert. Die zentralen Ergebnisse der Studie werden in Abschnitt 4 vorgestellt. Abschnitt 5 fasst den Beitrag zusammen und zieht ein Fazit.

\section{Verwandte Arbeiten}

In der Literatur existieren zahlreiche Richtlinien und Empfehlungen zur Ausgestaltung der Nachhaltigkeitsberichterstattung, beispielsweise von der Global Reporting Initiative (GRI 2006), der International Standards Organization (ISO 2006) oder dem World Business Council for Sustainable Development (WBCSD 2003). Ferner gibt es Rankings und Indizes, in denen Nachhaltigkeitsberichte von Unternehmen hinsichtlich ihrer Qualität untersucht und in eine Rangfolge gebracht wurden (bspw. IÖW2007). Ein Überblick über verwandte Arbeiten und ihre Analyse im Hinblick auf die nachfolgend aufgeführten Kriterien ist in Abbildung 1 gegeben.

- Qualitätskriterien: Präsentiert der Beitrag Prinzipien oder Kriterien für die Qualitätssicherung von Nachhaltigkeitsberichten (Ja (J), Nein(N))?

- Forschungs-)Methode: Auf der Basis welcher Forschungsmethodik werden Aussagen abgeleitet (A: Argumentativ-deduktiv; F: Fallstudien; E: Explorative/empirische Analyse)?

- Ergebnis: Was ist das zentrale Ergebnis des Beitrags (Empirische Befunde/Bestandsaufnahme (E), Referenzmodell (R), Qualitätskriterienkatalog (Q))?

- Fokus: Worauf fokussiert der Beitrag (Report-Inhalte (I), Report-Designkriterien (D), Report-Erstellungsprozess (E), Stakeholder-Perspektive (S), UnternehmensPerspektive (U))?

- Inhalt: Welche Inhalte werden in dem Beitrag diskutiert?

- Zentrale Erkenntnisse: Was sind die zentralen Erkenntnisse des Beitrags?

Im Gegensatz zu den genannten verwandten Arbeiten untersuchen die Autoren dieses Beitrags anhand von tatsächlich veröffentlichten Nachhaltigkeitsberichten, was der faktische Status Quo der Nachhaltigkeitsberichterstattung ist und welche Best Practices sich in der Unternehmenspraxis durchgesetzt haben. Unser Beitrag bietet somit die Möglichkeit, zu beurteilen, in wie weit eine Umsetzung der Empfehlungen und Richtlinien bereits in der betrieblichen Praxis erfolgt ist. 


\begin{tabular}{|c|c|c|c|c|c|}
\hline Quelle & 离 & 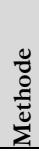 & 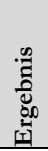 & $\frac{\mathscr{2}}{\vec{z}}$ & Inhalt/Zentrale Erkenntnisse \\
\hline \multirow[t]{2}{*}{$\begin{array}{l}\text { IÖW } \\
2007\end{array}$} & \multirow[t]{2}{*}{$\mathrm{J}$} & \multirow[t]{2}{*}{$\mathrm{E}$} & \multirow[t]{2}{*}{$\mathrm{E}$} & \multirow[t]{2}{*}{$\mathrm{I}$} & $\begin{array}{l}\text { Inhalt: Ranking der Nachhaltigkeitsberichte der } 150 \text { größten deutschen Unterneh- } \\
\text { men im Hinblick auf inhaltliche und kommunikative Qualität. }\end{array}$ \\
\hline & & & & & $\begin{array}{l}\text { Zentrale Erkenntnisse: } 58 \text { Unternehmen mit eigenständigem Bericht (insb. Che- } \\
\text { mie, Pharma, Banken) werden anhand eines Punktesystems zur Bewertung der } \\
\text { Qualität der Nachhaltigkeitsberichte in eine Rangfolge gebracht. } \\
\text { Entwicklungsbedarf in der Berichterstattung besteht insbesondere hinsichtlich der } \\
\text { sozialen Leistungsindikatoren; konkrete Maßnahmen zur Verbesserung der Indikato- } \\
\text { ren werden nur vereinzelt dargestellt. }\end{array}$ \\
\hline \multirow[t]{2}{*}{$\begin{array}{l}\text { IÖW } \\
2009\end{array}$} & \multirow[t]{2}{*}{$\mathrm{N}$} & \multirow[t]{2}{*}{$\mathrm{E}$} & \multirow[t]{2}{*}{$\mathrm{E}$} & \multirow[t]{2}{*}{$\mathrm{I}$} & $\begin{array}{l}\text { Inhalt: Unternehmensbefragung (61 Teilnehmer aus Grundgesamtheit der } 150 \\
\text { größten deutschen Unternehmen) zur Nachhaltigkeitsberichterstattung. }\end{array}$ \\
\hline & & & & & $\begin{array}{l}\text { Zentrale Erkenntnisse: Der Mix an medialen Formaten sowie die Ausrichtung der } \\
\text { Nachhaltigkeitsberichte an die individuellen Bedürfnisse der Stakeholder nimmt im } \\
\text { Vergleich zum Ranking aus } 2007 \text { (IÖW 2007) zu. }\end{array}$ \\
\hline \multirow[t]{2}{*}{$\begin{array}{l}\text { GRI } \\
2006\end{array}$} & \multirow[t]{2}{*}{$\mathrm{J}$} & \multirow[t]{2}{*}{$\mathrm{A}$} & \multirow[t]{2}{*}{$\begin{array}{l}\mathrm{R} \\
\mathrm{Q}\end{array}$} & \multirow[t]{2}{*}{$\begin{array}{l}\mathrm{I} \\
\mathrm{D}\end{array}$} & $\begin{array}{l}\text { Inhalt: Auf Basis eines Dialogs mit Stakeholdern der Wirtschaft, Anlegern, Arbeit- } \\
\text { geber- und Arbeitnehmervertretungen, der Zivilgesellschaft, Rechnungswesen, der } \\
\text { Wissenschaft und anderen Bereichen werden Empfehlungen zur Ausgestaltung der } \\
\text { Nachhaltigkeitsberichterstattung gegeben. }\end{array}$ \\
\hline & & & & & $\begin{array}{l}\text { Zentrale Erkenntnisse: } 13 \text { Qualitätskriterien an Nachhaltigkeitsberichte werden } \\
\text { aufgestellt, dabei werden jeweils auch Vorschläge für Testverfahren gemacht, mit } \\
\text { denen die Einhaltung dieser Kriterien überprüft werden kann. Weiterhin wird eine } \\
\text { Gliederung eines Nachhaltigkeitsberichts mit konkreten Inhalten und Kennzahlen als } \\
\text { Referenz vorgeschlagen. }\end{array}$ \\
\hline $\begin{array}{l}\text { ISO } \\
2006\end{array}$ & $\mathrm{~J}$ & A & $\begin{array}{l}\mathrm{R} \\
\mathrm{Q}\end{array}$ & $\mathrm{E}$ & $\begin{array}{l}\text { Inhalt / zentrale Erkenntnisse: Es werden } 5 \text { Qualitätsprinzipien zur Umwelt- } \\
\text { kommunikation von Unternehmen genannt und tabellarisch verschiedene Arten der } \\
\text { Umweltkommunikation gegenübergestellt, wobei die Erstellung eines Nachhaltig- } \\
\text { keitsberichtes nur eine mögliche Form ist. Es wird ein Referenz-Vorgehen zur } \\
\text { Umweltkommunikation beschrieben, ohne dabei auf konkrete Inhalte oder Kennzah- } \\
\text { len einzugehen. }\end{array}$ \\
\hline \multirow[t]{2}{*}{$\begin{array}{l}\text { KPMG } \\
2008\end{array}$} & \multirow[t]{2}{*}{$\mathrm{J}$} & \multirow[t]{2}{*}{$\mathrm{E}$} & \multirow[t]{2}{*}{$\mathrm{E}$} & \multirow[t]{2}{*}{$\mathrm{S}$} & $\begin{array}{l}\text { Inhalt: Auf der Basis von } 2279 \text { Teilnehmern weltweit wird aus Sicht der Leser von } \\
\text { Nachhaltigkeitsberichten (Stakeholder) untersucht, welche Anforderungen diese an } \\
\text { Inhalt und Qualität der Berichte haben. }\end{array}$ \\
\hline & & & & & $\begin{array}{l}\text { Zentrale Erkenntnisse: } 90 \% \text { der Studienteilnehmer konstatieren, dass durch das } \\
\text { Lesen der NBs sich die Sicht auf das Unternehmen geändert hat, davon hatten } 85 \% \\
\text { eine positivere Wahrnehmung des Unternehmens. Die Leser von Nachhaltigkeitsbe- } \\
\text { richten möchten verstärkt über konkrete Maßnahmen zur nachhaltigen Entwicklung } \\
\text { des Unternehmens informiert werden. }\end{array}$ \\
\hline $\begin{array}{l}\text { von } \\
\text { Ahsen et } \\
\text { al. } 2004\end{array}$ & $\mathrm{~N}$ & $\mathrm{E}$ & $\mathrm{E}$ & & $\begin{array}{l}\text { Inhalt / zentrale Erkenntnisse: Empirische Untersuchung sowie Befragung von } \\
63 \text { britischen und } 29 \text { Unternehmen aus Nordrhein-Westfalen mit Fokus auf Unter- } \\
\text { schiede in der Nachhaltigkeitsberichterstattung, die sich im Zuge der Umstellung von } \\
\text { EMAS I auf EMAS II ergeben haben. Untersucht werden die abgebildeten Umwelt- } \\
\text { aspekte sowie die Frage, warum Non-Reporter bisher keinen Nachhaltigkeitsbericht } \\
\text { veröffentlichen. }\end{array}$ \\
\hline $\begin{array}{l}\text { WBCSD } \\
2003\end{array}$ & $\mathrm{~J}$ & A & Q & $\begin{array}{l}\text { I } \\
\text { D } \\
\text { S }\end{array}$ & $\begin{array}{l}\text { Inhalt / zentrale Erkenntnisse: Der Ansatz des WBCSD ähnelt dem der GRI, } \\
\text { wobei die Vorgaben jedoch allgemeiner gehalten werden und eher Empfehlungscha- } \\
\text { rakter haben. Die Empfehlungen beziehen sich insbesondere auf die Informations- } \\
\text { bedürfnisse der Stakeholder sowie Berichtsinhalte. Um Non-Reporter zur Erstellung } \\
\text { eines Nachhaltigkeitsberichts zu motivieren, werden Zitate und Verweise auf beson- } \\
\text { ders erfolgreiche Unternehmen herangezogen. }\end{array}$ \\
\hline
\end{tabular}

\section{Abbildung 1: Verwandte Arbeiten}

Dabei sollen die Unternehmen nicht in eine Rangfolge gebracht, sondern vielmehr eine Rangfolge von referenzierten Standards und Richtlinien, verwendeten Technologien sowie berichteten Kennzahlen erstellt werden, so dass Praktiker in Form von Best Practices Verbesserungspotentiale für die eigene Nachhaltigkeitsbericht 
erstattung ableiten können. Der internationale Charakter unserer Studie erlaubt zudem das Feststellen von Gemeinsamkeiten und Unterschieden in der Nachhaltigkeitsberichterstattung der Regionen Deutschland, USA und Europa.

Ein zusätzlicher Schwerpunkt liegt auf der technischen Umsetzung der Nachhaltigkeitsberichte sowie den Interaktionsmöglichkeiten des Benutzers mit den Herausgebern des Nachhaltigkeitsberichts. Süpke, Gómez und Isenmann diskutieren in einem Beitrag (Süpke et al. 2009) die Einsatzmöglichkeiten von Web 2.0Technologien zur Kommunikation über Nachhaltigkeitsberichte zwischen Stakeholdern, zwischen Unternehmen und Stakeholdern sowie unternehmensintern. Wir untersuchen daher mit unserem Beitrag, in wie weit diese innovativen Technologien bereits in der Praxis eingesetzt werden.

\section{Analyse-Rahmenwerk und Methodik}

Im Rahmen dieses Beitrags wurden von den Autoren die Nachhaltigkeitsberichte von 97 Unternehmen systematisch untersucht. Auf Basis der Annahme, dass insbesondere börsennotierte Unternehmen Nachhaltigkeitsberichte veröffentlichen und unter der Prämisse, dass ein Ländervergleich ermöglicht werden soll, wurden der Deutsche Aktienindex (DAX, 30 Unternehmen), der Dow Jones Industrial Average Index (Dow Jones, 30 Unternehmen) sowie der Dow Jonex Euro Stoxx 50 Index (EuroStoxx, 50 Unternehmen) ausgewählt. Um Doppelzählungen und damit Verfälschungen bei den Auswertungen zu vermeiden, wurden dabei die 13 im EuroStoxx enthaltenen DAX-Unternehmen bei den Auswertungen zu den EuroStoxx-Unternehmen nicht berücksichtigt.

Die auszuwertenden Merkmale wurden im Rahmen eines Workshops erarbeitet; die Datenerhebung erfolgte im Laufe des Septembers 2009 anhand der aktuell auf den Webseiten der jeweiligen Unternehmen veröffentlichten Nachhaltigkeitsberichte. Da es bei der Zuordnung der Nachhaltigkeitsberichte zu den Ausprägungen der Analysevariablen gewisse Freiheitsgrade und subjektiven Spielraum gibt, wurden insgesamt 3 Personen an der Entwicklung der Vorgehensweise, der Auswahl der Analysevariablen sowie der Durchführung der eigentlichen Analyse beteiligt.

Die Eingruppierungen wurden zunächst von einer Person durchgeführt und anschließend durch 2 weitere Personen stichprobenhaft überprüft. Hierbei lagen die Übereinstimmungen bei den einzelnen Analysevariablen bei jeweils mehr als 90\%. Die übrig gebliebenen unstimmigen Zuordnungen wurden im Rahmen einer gemeinsamen Diskussion ausgeräumt.

Abbildung 2 zeigt das Analyse-Rahmenwerk, das bei der Erstellung dieses Beitrags zu Grunde gelegt wurde 
Die Abbildung gibt einen Überblick über die verschiedenen Forschungsphasen und deren zentrale Forschungsfragen. In der Spalte Abschnitt ist angegeben, in welchen Abschnitten dieses Beitrags die Ergebnisse aus der jeweiligen Phase zu finden sind. Trends und regionale Unterschiede in der Nachhaltigkeitsberichterstattung, Implikationen für Wissenschaft, Praxis und zukünftige Forschungsarbeiten sind Untersuchungsgegenstand aller vier Phasen.

\begin{tabular}{|l|l|l|l|}
\hline Phase & Stufe & Forschungsfrage/Inhalt & $\begin{array}{l}\text { Ab- } \\
\text { schnitt }\end{array}$ \\
\hline $\begin{array}{l}\text { Motivation/ } \\
\text { Allgemein }\end{array}$ & 1. Warum? & $\begin{array}{l}\text { Warum Nachhaltigkeitsberichterstattung? Welche } \\
\text { verwandten Arbeiten gibt es? }\end{array}$ & $1,2,4.1$ \\
\hline Inhalte & 2. Was? & $\begin{array}{l}\text { Was sind die Inhalte der Nachhaltigkeitsberichter- } \\
\text { stattung? }\end{array}$ & 4.2 \\
\hline $\begin{array}{l}\text { Technik/ } \\
\text { Organisation }\end{array}$ & 3. Wie? & $\begin{array}{l}\text { Wie ist die Berichterstattung technisch umgesetzt? } \\
\text { Welche Kommunikationsmöglichkeiten werden } \\
\text { geboten? }\end{array}$ & 4.3 \\
\hline $\begin{array}{l}\text { Richtlinien } \\
\text { und Ran- } \\
\text { kings }\end{array}$ & 4. Woran? & $\begin{array}{l}\text { Woran orientieren sich Unternehmen bei ihrer } \\
\text { Nachhaltigkeitsberichterstattung (Richtlinien, Zerti- } \\
\text { fizierungen, Rankings und Indizes)? }\end{array}$ & 4.4 \\
\hline
\end{tabular}

\section{Abbildung 2: Analyse- Rahmenwerk}

\section{Ergebnisse}

Im Folgenden werden die Ergebnisse der Untersuchung vorgestellt. Aus Platzgründen werden die untersuchten Merkmale sowie die gewonnenen Erkenntnisse teilweise kurz in Tabellenform zusammengefasst.

\subsection{Motivation / Allgemein}

Angesichts des hohen Anteils von berichterstattenden Unternehmen (4.1.1), sehen sich die Autoren in ihrer grundsätzlichen Annahme, dass insbesondere börsennotierte Unternehmen Nachhaltigkeitsberichte veröffentlichen, bestätigt.

\begin{tabular}{|l|l|}
\hline Untersuchungsmerkmal & Erläuterung \\
\hline 4.1.1 Aktueller Bericht? & $\begin{array}{l}\text { Prozentualer Anteil der Unternehmen, die einen aktuellen Nachhaltigkeitsbe- } \\
\text { richt (nicht älter als 2006) veröffentlichen. }\end{array}$ \\
\hline 4.1.2 Link auf Startseite & $\begin{array}{l}\text { Prozentualer Anteil der Unternehmen, die einen direkten Link zum Nachhaltig- } \\
\text { keitsbericht auf ihrer Startseite haben. }\end{array}$ \\
\hline $\begin{array}{l}\text { 4.1.3 Aktualität der Berich- } \\
\text { te }\end{array}$ & $\begin{array}{l}\text { Prozentuale Untersuchung der Jahreszahlen, aus denen der jeweils aktuellste } \\
\text { Nachhaltigkeitsbericht der Unternehmen stammt. }\end{array}$ \\
\hline 4.1.4 Historie & $\begin{array}{l}\text { Untersuchung, wie viele Unternehmen (Anzahl) je Jahr erstmalig einen Nachhal- } \\
\text { tigkeitsbericht veröffentlicht haben. Die Angaben stammen aus den Nachhaltig- } \\
\text { keitsberichten der Unternehmen oder wurden anhand eines ggf. vorhandenen } \\
\text { Berichtsarchivs ermittelt. }\end{array}$ \\
\hline $\begin{array}{l}\text { 4.1.5 Top-Management } \\
\text { Support }\end{array}$ & $\begin{array}{l}\text { Prozentuale Untersuchung des jeweils aktuellsten Berichts der Unternehmen, ob } \\
\text { ein hochrangiger Vertreter des Unternehmens (Vorstand, Geschäftsführer, etc.) } \\
\text { sein Nachhaltigkeits-Commitment zum Ausdruck bringt. }\end{array}$ \\
\hline
\end{tabular}

Abbildung 3: Untersuchungsmerkmale Motivation/Allgemein 


\begin{tabular}{|c|c|c|c|c|c|}
\hline & \multicolumn{2}{|c|}{ Untersuchungsmerkmal } & DAX & Dow & EuroStoxx \\
\hline \multirow{6}{*}{ 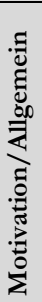 } & \multicolumn{2}{|c|}{ 4.1.1 Aktueller Bericht? } & $83,33 \%$ & $93,33 \%$ & $94,59 \%$ \\
\hline & \multicolumn{2}{|c|}{ 4.1.2 Link auf Startseite? } & $63,33 \%$ & $46,67 \%$ & $81,08 \%$ \\
\hline & \multirow[t]{3}{*}{ 4.1.3 Aktualität } & 2009 & $24 \%$ & $25 \%$ & $2,86 \%$ \\
\hline & & 2008 & $68 \%$ & $71,43 \%$ & $94,29 \%$ \\
\hline & & 2007 & $8 \%$ & $3,57 \%$ & $2,86 \%$ \\
\hline & \multicolumn{2}{|c|}{ 4.1.5 Top-Management-Support } & $96,15 \%$ & $89,29 \%$ & $88,57 \%$ \\
\hline
\end{tabular}

Abbildung 4: Auswertung Motivation/Allgemein

Im Vergleich des Anteils der Unternehmen, die einen direkten Link zum Nachhaltigkeitsbericht auf ihrer Startseite haben (4.1.2), ergeben sich deutliche Unterschiede. Insbesondere amerikanische Unternehmen veröffentlichen den Nachhaltigkeitsbericht häufig in einem Unterpunkt der „About us“-Sektion auf der Firmenhomepage und nicht direkt auf der Startseite. Eventuell soll dadurch erreicht werden, dass nur Besucher, die gezielt nach Nachhaltigkeitsinformationen suchen, den Nachhaltigkeitsbericht aufrufen. Nach den Erkenntnissen der Studie von KPMG (KPMG 2008 S. 10) verspielen dadurch viele Unternehmen die Möglichkeit, ihre Wahrnehmung durch Stakeholder positiv durch einen Nachhaltigkeitsbericht zu beeinflussen.

Beim Vergleich der Aktualität der Nachhaltigkeitsberichte zeigt sich, dass insbesondere in den USA und Deutschland auch unterjährig Zwischenberichte veröffentlicht werden. Teilweise ist diese Beobachtung auch dadurch zu begründen, dass der Reporting-Zyklus einiger Unternehmen sich nicht am Kalenderjahr, sondern am Geschäftsjahr orientiert. Nahezu alle Unternehmen veröffentlichen Nachhaltigkeitsberichte, die maximal aus dem vergangenen Jahr stammen.

Abbildung 5 gibt einen Überblick über die Historie der Nachhaltigkeitsberichte (4.1.4).

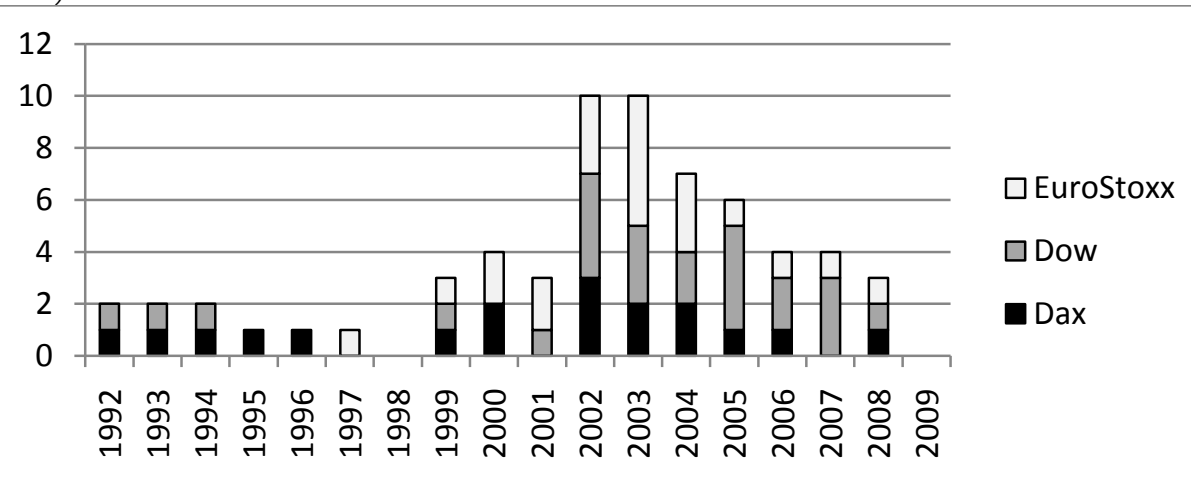

Abbildung 5: Anzahl der erstmalig reportenden Unternehmen 
In den Jahren 1992-1996 gab es in den USA und Deutschland bereits einige Early Adopters, die einen Nachhaltigkeitsbericht veröffentlicht haben - im restlichen Europa setzt die Entwicklung erst deutlich später ein, wird durch höhere Wachstumsraten jedoch wieder aufgeholt. Dabei ist zu berücksichtigen, dass die frühen Nachhaltigkeitsberichte in Umfang und Qualität nicht an aktuelle Berichte heranreichen. Auffällig ist, dass im Jahr 2000 in den USA bzw. im Jahr 2001 in Deutschland keine neuen Unternehmen hinzugekommen sind. Man könnte hier vor dem Hintergrund des Börsencrashs um die Jahrtausendwende spekulieren, ob eine Wirtschaftskrise evtl. eine Nachhaltigkeitskrise nach sich gezogen hat. Besonders viele Erstreporter finden sich in den Jahren 2002-2005; hier spielt vermutlich das gesteigerte regulatorische und öffentliche Interesse an Umweltdaten eine entscheidende Rolle. In den letzten Jahren sinken die Wachstumsraten, da nahezu alle Unternehmen bereits einen Nachhaltigkeitsbericht veröffentlichen.

Die Untersuchung des Top-Management Supports (4.1.5) zeigt, dass ein TopManagement-Commitment für die Nachhaltigkeit im Rahmen eines NBs heutzutage ein De-facto-Standard ist.

\subsection{Inhalte}

Die Auswertung der Nachhaltigkeitsaspekte (4.2.1) zeigt, dass alle drei Säulen der Nachhaltigkeit größtenteils von den Berichten abgedeckt werden. Im Bereich der Verbräuche und insbesondere im Bereich der Abfälle, bestehen in den USA und Europa jedoch noch Berichtslücken. Eventuell behalten Unternehmen diese Informationen gezielt für sich, um der Konkurrenz keine Rückschlüsse auf die Ausgestaltung der Produktionsverfahren und deren Effizienz zu erlauben.

\begin{tabular}{|l|l|}
\hline Untersuchungsmerkmal & Erläuterung \\
\hline $\begin{array}{l}\text { 4.2.1 Nachhaltigkeitsas- } \\
\text { pekte }\end{array}$ & $\begin{array}{l}\text { Untersuchung, ob der Nachhaltigkeitsbericht ökonomische, ökologische } \\
\text { und soziale Aspekte abdeckt. Die ökologischen Aspekte werden dabei } \\
\text { zusätzlich in Emissionen, Verbräuche und Abfälle unterteilt. }\end{array}$ \\
\hline 4.2.2 Kennzahlen & $\begin{array}{l}\text { Prozentualer Anteil von Berichten, in denen eine bestimmte Kennzahl } \\
\text { verwendet wurde. Zunächst wurde eine größere Anzahl von Kennzahlen } \\
\text { untersucht, nur vereinzelt genannte wurden in einem zweiten Schritt ent- } \\
\text { fernt, um die Übersichtlichkeit zu erhöhen. }\end{array}$ \\
\hline $\begin{array}{l}\text { 4.2.3 Richtlinien, Zertifi- } \\
\text { zierungen und Rankings } \\
\text { (Indizes) }\end{array}$ & $\begin{array}{l}\text { Prozentualer Anteil von Berichten, in denen auf eine bestimmte Richtlinie, } \\
\text { Zertifizierung oder ein Ranking (Index) verwiesen wird. Analog zum Vor- } \\
\text { gehen bei den Kennzahlen wurde zunächst eine höhere Anzahl untersucht } \\
\text { und in einem zweiten Schritt eine Einschränkung vorgenommen. }\end{array}$ \\
\hline 4.2.4 Seitenzahl & $\begin{array}{l}\text { Untersuchung der minimalen, maximalen und durchschnittlichen Seitenzahl } \\
\text { der Nachhaltigkeitsberichte je Branche. }\end{array}$ \\
\hline
\end{tabular}

Abbildung 6: Untersuchungsmerkmale Inhalt 


\begin{tabular}{|c|c|c|c|c|c|c|}
\hline & \multicolumn{3}{|l|}{ Untersuchungsmerkmal } & DAX & Dow & EuroStoxx \\
\hline \multirow{5}{*}{ 壹 } & \multirow{5}{*}{$\begin{array}{l}4.2 .1 \text { Nachhaltigkeitsas- } \\
\text { pekte }\end{array}$} & \multicolumn{2}{|l|}{ Ökonomisch } & $92 \%$ & $96,43 \%$ & $100 \%$ \\
\hline & & \multirow[t]{3}{*}{ Ökologisch } & Emissionen & $96 \%$ & $96,43 \%$ & $97,14 \%$ \\
\hline & & & Verbräuche & $100 \%$ & $85,71 \%$ & $97,14 \%$ \\
\hline & & & Abfälle & $88 \%$ & $67,86 \%$ & $74,29 \%$ \\
\hline & & \multicolumn{2}{|c|}{ Gesellschaft/Soziales } & $100 \%$ & $92,86 \%$ & $100 \%$ \\
\hline
\end{tabular}

\section{Abbildung 7: Auswertung Nachhaltigkeitsaspekte}

Abbildung 8 gibt einen Überblick über die Häufigkeit der Verwendung von Kennzahlen (4.2.2).

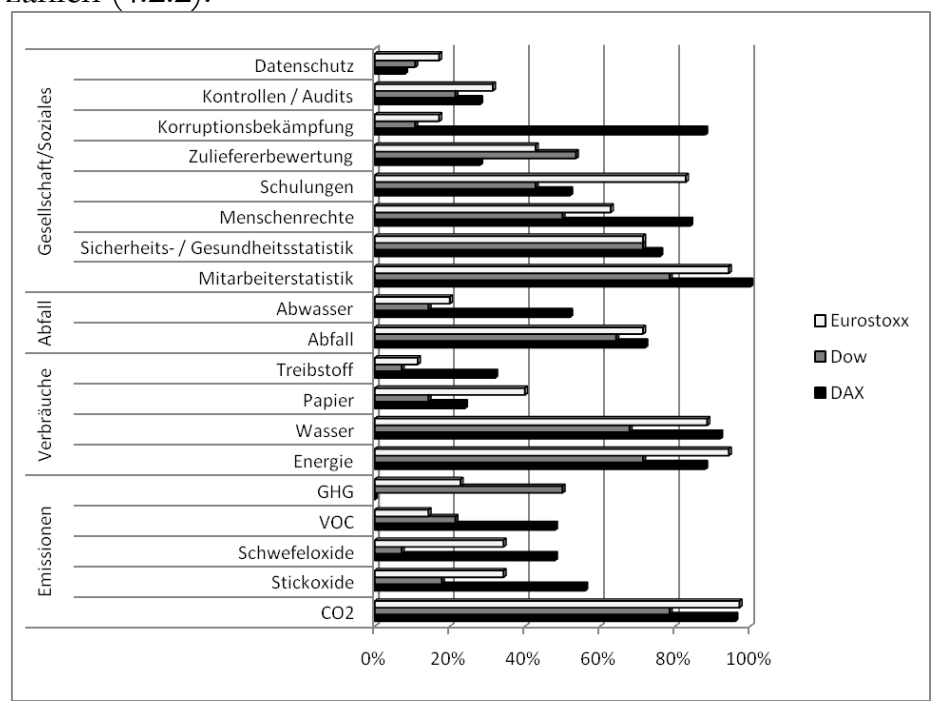

\section{Abbildung 8: Häufigkeit von Kennzahlen}

Im Bereich der Emissionen dominiert die Kennzahl CO2-Ausstoß. Der Ausweis der Stick- und Schwefeloxid- sowie VOC-Emissionen (Volatile Organic Air) ist in den USA nicht so verbreitet wie in Deutschland und Europa, dafür erfolgt in den USA häufig der allgemeiner gefasste Ausweis der Treibhausgas-Emissionen (GHG). Diese Beobachtung bestätigt die subjektive Wahrnehmung der Autoren, dass die deutschen Nachhaltigkeitsberichte in der Regel detaillierter als in den übrigen Regionen sind. In amerikanischen Nachhaltigkeitsberichten finden sich häufig längere Textpassagen um allgemein gehaltene Kennzahlen, während in deutschen Nachhaltigkeitsberichten häufiger ,hartes' Datenmaterial in Form von Tabellen bereitgestellt wird.

Im Bereich der Verbräuche wird in nahezu allen Unternehmen der Energieund Wasserverbrauch ausgewiesen. Der Verbrauch von Papier und Treibstoff ist hingegen nicht für alle Unternehmen relevant. Die Berichtslücken im Bereich Ab- 
fall wurden bereits diskutiert. In dieser Abbildung ist zusätzlich auffällig, dass in Deutschland doppelt so häufig wie in den übrigen Regionen Angaben über entstandenes Abwasser gemacht werden. Im Bereich Gesellschaft/Soziales gehören eine Mitarbeiter- sowie eine Sicherheits- bzw. Gesundheitsstatistik zum Standard. In Deutschland werden etwa vier Mal so häufig Angaben zur Korruptionsbekämpfung gemacht, wie im Rest der Welt.

Abbildung 9 gibt einen Überblick über die Häufigkeit des Verweises auf bestimmte Richtlinien, Zertifizierungen und Rankings (4.2.3) im Bereich der Nachhaltigkeit.

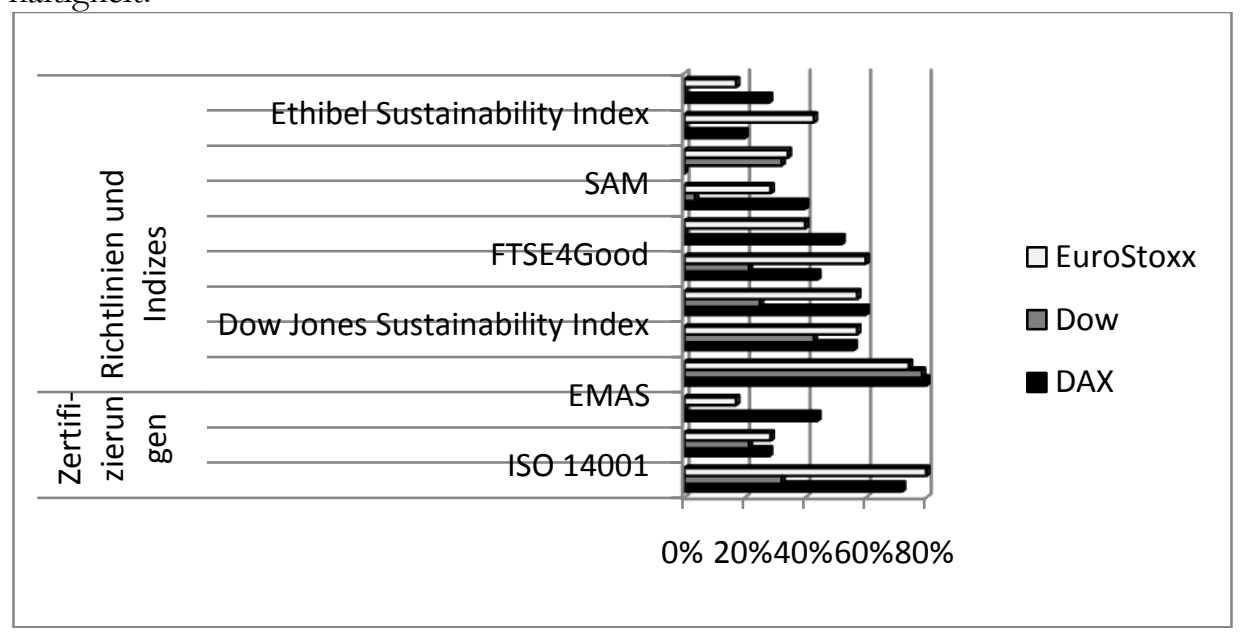

\section{Abbildung 9: Häufigkeit von Zertifizierungen, Richtlinien und Rankings}

Die Auswertung zeigt, dass Zertifizierungen im Bereich Umwelt in Deutschland und Europa eine deutlich größere Rolle als in den USA spielen. Wenig überraschend spielt die EMAS-Zertifizierung, die nur für europäische Unternehmen vorgesehen ist, in den USA keine Rolle. Hinsichtlich der Richtlinien nehmen etwa $80 \%$ der Unternehmen unabhängig von der Region Bezug auf die GRI (GRI 2006). Aus Sicht der Leser von Nachhaltigkeitsberichten ist dies erfreulich, da die Studie von KPMG (KPMG 2008 S. 13) ergeben hat, dass die GRI auch aus Lesersicht der wichtigste Standard zur Nachhaltigkeitsberichterstattung sind.

Obwohl der Seitenumfang allein kein inhaltliches Qualitätsmerkmal für einen Nachhaltigkeitsbericht sein kann (IÖW 2007 S.2), wurde für deutsche Unternehmen in Abhängigkeit von der Unternehmensbranche untersucht, wie hoch der Seitenumfang der Nachhaltigkeitsberichte ist (4.24). Aus Platzgründen werden an dieser Stelle lediglich die wesentlichen Erkenntnisse zusammengefasst. Es zeigt sich, dass insbesondere in den Branchen Banken/Finanzen/Versicherungen sowie Chemie/Pharma der Seitenumfang zwischen den Unternehmen stark schwankt. Generell lässt sich jedoch die Tendenz beobachten, dass stark umweltverschmutzende Branchen wie Chemie/Pharma, Energieversorger, Transport/Logistik und die Automobilindustrie besonders umfassende Nachhaltigkeitsberichte veröffentli- 
chen. Bei einem durchschnittlichen Seitenumfang von 142 Seiten in der Chemieund Pharmaindustrie wird deutlich, warum Wissenschaftler zunehmend Konzepte für Nachhaltigkeitsberichte entwickeln, die dynamisch nach den Anforderungen des jeweiligen Stakeholders generiert werden (Isenmann und Gómez 2008).

\subsection{Technik}

Länderübergreifend sind Webseiten oder Berichte im drucknahen PDF-Format am beliebtesten. In Deutschland und Europa werden geringfügig mehr Webseiten als PDF-Berichte eingesetzt, in den USA umgekehrt. Technisch aufwändige OnlinePortale haben in den USA 3 von 4 Unternehmen bereits umgesetzt, hier haben Europa und insbesondere Deutschland noch Aufholbedarf. Die Bereitstellung von Daten im Excel-Format, so dass Anwender die Daten selbst auswerten und weiterverarbeiten können, ist eine deutsche Domäne - in Europa bzw. den USA ist dies nicht üblich. Diese Beobachtung stärkt erneut den Eindruck der Autoren, dass deutsche Nachhaltigkeitsberichte kennzahlenlastiger als in den übrigen Regionen sind.

Hinsichtlich der Feedbackmöglichkeiten (4.3.2) zeigt sich, dass Kommunikation überwiegend per E-Mail stattfindet. Kontaktformulare werden in einem kleinen Teil der Unternehmen in den USA und Deutschland angeboten, Newsletter sind in den USA am weitesten verbreitet. Umfragen sind in den USA und Deutschland ebenfalls ein relativ beliebtes Kommunikationsmittel. Kein Unternehmen ermöglicht es den Benutzern, öffentlich sichtbare Kommentare oder Trackbacks zum Nachhaltigkeitsbericht abzugeben.

\begin{tabular}{|l|l|}
\hline Untersuchungsmerkmal & Erläuterung \\
\hline 4.3.1 Format & $\begin{array}{l}\text { Untersuchung, in welchem Format die Nachhaltigkeitsberichte bereitge- } \\
\text { stellt werden. Unterschieden wird zwischen Nachhaltigkeitswebseiten, die } \\
\text { in die Unternehmenshomepage integriert sind, eigenständigen Webporta- } \\
\text { len, statischen Dokumenten (PDF etc.) sowie der Möglichkeit, Informatio- } \\
\text { nen im Excel-Format zur weiteren Nutzung herunterzuladen. Mehrfach- } \\
\text { nennungen sind möglich. }\end{array}$ \\
\hline 4.3.2 Feedback & $\begin{array}{l}\text { Untersuchung, in wie weit der Nutzer des Nachhaltigkeitsberichts in den } \\
\text { Dialog mit dem Unternehmen oder auch mit anderen Nutzern treten kann. }\end{array}$ \\
\hline 4.3.3 Organisation & $\begin{array}{l}\text { Untersuchung, welche Unternehmensbereiche für die Erstellung des Nach- } \\
\text { haltigkeitsberichts verantwortlich sind. }\end{array}$ \\
\hline
\end{tabular}

\section{Abbildung 10: Untersuchungsmerkmale Technik / Organisation}

Dies könnte eventuell in der Angst vor negativen Kommentaren und dem daraus folgenden Rechtfertigungsbedarf begründet sein. Innovative Web 2.0 Technologien, die eine bidirektionale Kommunikation ermöglichen, werden demnach noch zu wenig genutzt. Hier ist die Wissenschaft gefordert, zum einen die technologi- 
schen Grundlagen weiterzuentwickeln, und zum anderen zu untersuchen, in wie weit die Ängste der Unternehmen vor negativem Feedback begründet sind.

Bezüglich der Verantwortlichkeit für den Nachhaltigkeitsbericht (4.3.3) zeigt sich, dass neben der Umweltabteilung häufig auch die Abteilung für Öffentlichkeitsarbeit oder externe Dienstleister und Beratungsunternehmen in den Erstellungsprozess eingebunden werden.

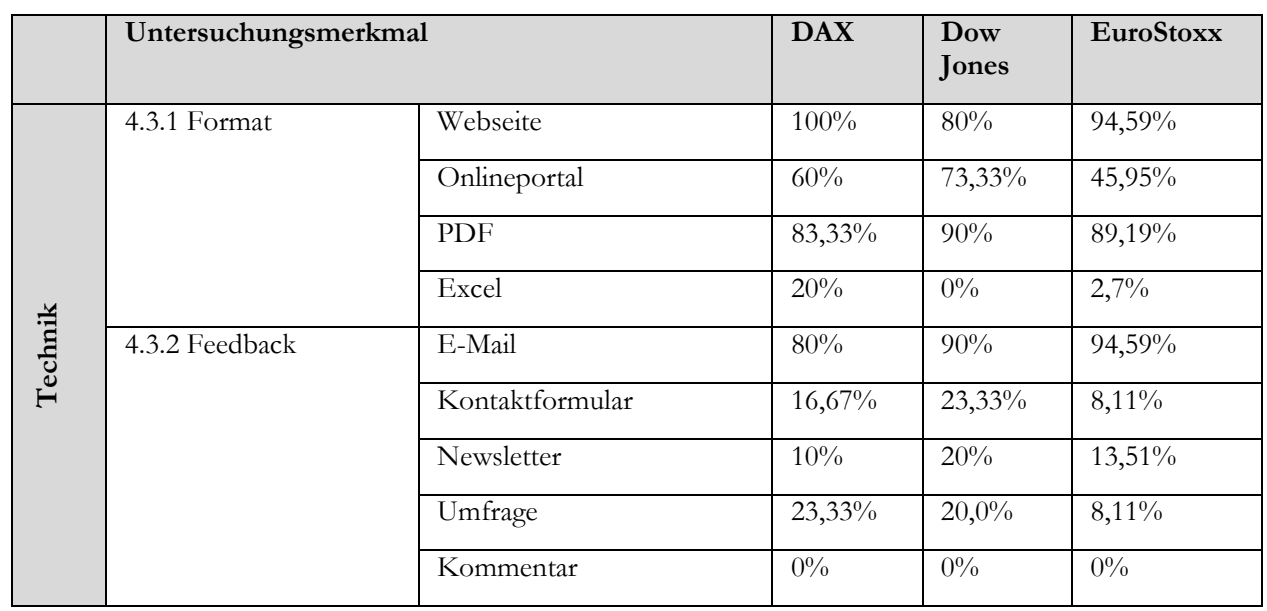

Abbildung 11: Auswertung Technik

\section{Zusammenfassung und Ausblick}

Im Rahmen dieses Beitrags wurden die veröffentlichten Nachhaltigkeitsberichte von 97 börsennotierten Unternehmen aus Deutschland, dem restlichen Europa sowie den USA quantitativ untersucht. Da im Internet eine unüberschaubare Anzahl von Nachhaltigkeitsberichten verfügbar ist, musste zur Durchführung dieser Studie eine Auswahl getroffen werden. Dabei sind die Autoren von der Annahme, dass insbesondere börsennotierte Unternehmen Nachhaltigkeitsberichte veröffentlichen, systematisch anhand von Börsenindizes vorgegangen. Die erzielten Ergebnisse können somit nicht repräsentativ für alle Unternehmen sein, bieten jedoch einen vollständigen Überblick über die Unternehmen in den jeweiligen Indizes, und somit auch für andere Unternehmen wichtige Erkenntnisse über Best Practices der Nachhaltigkeitsberichterstattung. Auch kleine und mittlere Unternehmen (KMU), die eventuell noch keinen Nachhaltigkeitsbericht veröffentlichen, können so von den Erfahrungen börsennotierter Großunternehmen profitieren, indem sie ihre Berichtsinhalte, angestrebte Zertifizierungen und zu berücksichtigende Richtlinien an den Best Practices der börsennotierten Unternehmen orientieren. Auch hinsichtlich der technologischen Basis sowie organisatorischer Verantwortlichkeiten bietet unser Beitrag eine erste Orientierungshilfe für Praktiker. 
Aus Sicht der Wissenschaft zeigt sich, dass in vielen Bereichen der Nachhaltigkeitsberichterstattung bereits ein recht hohes Niveau erreicht ist, und dass sich erste De-Facto-Standards herauskristallisiert haben. Forschungsbedarf besteht angesichts des teilweise hohen Seitenumfangs der Nachhaltigkeitsberichte insbesondere hinsichtlich einer flexiblen Anpassung der Nachhaltigkeitsberichte an die Informationsbedürfnisse der Stakeholder(-gruppen), sowie hinsichtlich des Einsatzes von Web 2.0-Technologien zur bidirektionalen Kommunikation zwischen Unternehmen und Stakeholdern. Erste Vorarbeiten zu diesen Gebieten bestehen bereits (Isenmann und Gómez 2008; Süpke et al. 2009), müssen sich in der Praxis jedoch noch bewähren und durchsetzen.

Nachdem in diesem Beitrag börsennotierte Unternehmen betrachtet wurden, bietet sich zukünftig die Untersuchung von KMUs an, da hier vermutlich noch ein geringerer Reifegrad in der Nachhaltigkeitsberichterstattung und damit Aufholbedarf im Vergleich zu börsennotierten Unternehmen besteht. Auch regionale oder branchenabhängige Unterschiede könnten deutlicher als bei den börsennotierten Unternehmen ausfallen und damit einen interessanten Ansatzpunkt für weitere Forschung bieten.

Die Autoren sind der Meinung, dass im Bereich der qualitativen Analyse bestehender Nachhaltigkeitsberichte noch erhebliches Erkenntnispotential über den Status Quo, Branchenunterschiede und Veränderungen der inhaltlichen Schwerpunkte im Zeitablauf besteht. Denkbar wäre beispielsweise die softwareunterstütze Zählung und Visualisierung von Schlagwortnennungen, um diese Unterschiede ohne Untersucher-Bias herauszuarbeiten.

Aufgrund des Stakeholder-Fokus besteht hinsichtlich der Usability von Nachhaltigkeitsberichten, die in Form von Interviews, Befragungen oder Experimenten (Eye Tracking etc.) untersucht werden könnte, ebenfalls weiterer Forschungsbedarf.

\section{Literatur}

GRI (2006) Sustainability Reporting Guidelines.

http://www.globalreporting.org/ReportingFramework/ReportingFramework Downloads/. Abruf am 2009-10-04.

Günther E (1988) Ökologieorientiertes Management. UTB, Stuttgart.

IÖW (2007) Nachhaltigkeitsberichterstattung in Deutschland: Ergebnisse und Trends im Ranking 2007. http:/ /www.rankingnachhaltigkeitsberichte.de/2_3ergebnisse.html. Abruf am 2009-10-04.

IÖW (2009) Praxis der Nachhaltigkeitsberichterstattung in deutschen Grossunternehmen. http://www.ranking-nachhaltigkeitsberichte.de/. Abruf am 2009-10-04.

Isenmann R, Gómez JM (2008) Internetbasierte Nachhaltigkeitsberichterstattung Maßgeschneiderte Stakeholder-Kommunikation mit IT. Schmidt, Berlin. 
ISO (2006) ISO 14063 - Environmental Management - Environmental Communication - Guidelines and Examples. http://www.iso.org/iso/iso_catalogue/catalogue_tc/catalogue_detail.htm?csn umber=34676. Abruf am 2009-10-04.

KPMG (2008) Count me in - The readers' take on sustainability reporting. http://www.globalreporting.org/CurrentPriorities/GlobalReadersSurvey/. Abruf am 2009-10-04.

Süpke D, Gómez JM, Isenmann R (2009) Web 2.0 for Sustainability Reporting Approach to Refining Communication on Sustainability. In: Wohlgemuth V, Page B, Voigt K (Hrsg) Proceedings of the $23^{\text {rd }}$ International Conference on Informatics for Environmental Protection, Berlin: 219-228.

Von Ahsen A., Lange C., Pianowski M. (2004) Corporate Environmental Reporting - Survey and Empirical Evidence. International Journal of Environment and Sustainable Development 3(1):5-17.

WBCSD (2003) Sustainable Development Reporting. http://www.wbcsd.org/. Abruf am 2009-10-04. 